\title{
Downregulated expression of microRNAs associated with cardiac hypertrophy and fibrosis in physiological pregnancy and the association with echocardiographically-evaluated myocardial function
}

\author{
EWA SZCZERBA ${ }^{1,2}$, AGNIESZKA ZAJKOWSKA ${ }^{3}$, ANNA BOCHOWICZ ${ }^{1}$, \\ KATARZYNA PANKIEWICZ ${ }^{4}$, GRZEGORZ SZEWCZYK ${ }^{4}$, GRZEGORZ OPOLSKI ${ }^{2}$, \\ TOMASZ MACIEJEWSKI ${ }^{4}$, MACIEJ MAŁECKI $^{3}$ and ANNA FIJAŁKOWSKA ${ }^{1}$ \\ ${ }^{1}$ Department of Cardiology, Institute of Mother and Child, 01-211 Warsaw; ${ }^{2}$ First Chair and Department of Cardiology, \\ ${ }^{3}$ Department of Applied Pharmacy, Medical University of Warsaw, 02-097 Warsaw; \\ ${ }^{4}$ Department of Gynecology and Obstetrics, Institute of Mother and Child, 01-211 Warsaw, Poland
}

Received November 16, 2019; Accepted July 15, 2020

DOI: $10.3892 /$ br.2020.1348

\begin{abstract}
The aim of the present study was to analyze the profiles of cardiac microRNAs (miRNAs/miRs) in healthy pregnant women and non-pregnant controls. A total of 61 healthy women $>18$ years of age with singleton pregnancies in the third trimester were compared with 19 non-pregnant controls. Specifically, expression of miRNAs associated with cardiac hypertrophy (miR-1, miR-17-5, miR-22, miR-34a, miR-124, miR-133a, miR-195, miR-199a-3p, miR-199b, miR-210, miR-222 and miR-1249) and miRNAs associated with cardiac hypertrophy and fibrosis (miR-15b, miR-21, miR-26a, miR-29-a, miR-29c, miR-30c, miR-101, miR-146a, miR-191, miR-208a-5p and miR-328) were analyzed and compared with echocardiographic examination results. Both groups had similar cardiac miRNA expression profiles, but differed in quantitative evaluation. Women in the third trimester of physiological pregnancy exhibited downregulation of certain profibrotic miRNAs (miR-21, miR-30c and miR-328), decreased expression of a hypertrophic and antimetabolic miRNAs (miR-146a), downregulation of an antifibrotic miRNA (miR-222), and downregulation of a hypertrophic miRNA (miR-195). In pregnant women, the indices of systolic function were associated with miR-195 expression, and an interplay between miR-17-5p and diastolic function was observed. While the profiles of cardiac miRNAs expressed in healthy pregnant women and healthy non-pregnant controls
\end{abstract}

Correspondence to: Professor Anna Fijałkowska, Department of Cardiology, Institute of Mother and Child, Kasprzaka 17a Street, 01-211 Warsaw, Poland

E-mail: anna.fijalkowska@imid.med.pl

Key words: cardiac adaptation, volume overload, pregnancy, remodeling, microRNA, hypertrophy were similar, these two groups differed in terms of expression of specific miRNAs. In the third trimester of physiological pregnancy, a downregulation of miR-17-5p, miR-21, miR-30c, miR-146a, miR-195, miR-222 and miR-328 was observed. The differences in the association between echocardiographic indices with miRNAs in pregnant and non-pregnant women suggest that miRNAs regulate both the structure and function of the pregnant heart, influencing cardiac muscle thickness as well as systolic and diastolic function.

\section{Introduction}

During pregnancy, the maternal cardiovascular system is subjected to volume overload (1). However, pressure overload is not observed unless the pregnancy is complicated by gestational hypertension (1). The increase in blood volume activates several cardiac adaptive mechanisms, which results in the enlargement of the left and right heart chambers and an increase in cardiac mass (1). The most evident increase in volume overload occurs during the third trimester (2). The changes that take place during physiological pregnancy are a form of physiological reversible hypertrophy. Alterations in the diastolic function and contractility have been described previously $(3,4)$. For example, lack of fibrosis accompanying the hypertrophy allows the cardiac muscle to maintain or even increase its lusitropic capacities.

The underlying molecular mechanisms of the changes to cardiac structure and function are not fully understood (5). An emerging mediator involved in the adaptive processes are microRNAs (miRNAs/miRs). Several miRNAs have been linked to cardiovascular diseases, and even proposed as cardiovascular biomarkers (6). Furthermore, miRNAs have been shown to be involved in the adaptation of the cardiovascular system to exercise, another situation which where physiological hypertrophy is observed (7).

Studies of the role of miRNAs in cardiovascular alterations associated with pregnancy is limited primarily to 
preeclampsia $(8,9)$. Additionally, the potential involvement of miRNAs in peripartum cardiomyopathy is the subject of only a limited number of published studies $(10,11)$. As miRNAs regulate gene expression under specific conditions influencing cellular activity, it was hypothesized that they may serve a crucial role in cardiac adaptation to pregnancy-related volume overload (12). MicroRNAs associated with stimulation or inhibition of cardiac hypertrophy and fibrosis were chosen for further inspection.

Detailed information regarding the expression of cardiac miRNAs in pregnancy may improve our understanding of the physiological adaptations which take place to accommodate volume overload, and additionally may serve as a reference for various pathological conditions, for example dilated cardiomyopathy. The aim of the present study was to analyze the profile of miRNAs associated with cardiac hypertrophy and fibrosis during the period of maximum volume overload. To identify potential pregnancy-specific changes in the expression of cardiac miRNAs, the results obtained in pregnant women were compared with those from non-pregnant healthy controls.

The overall hypothesis of the present study was that there is a difference in the expression of cardiac miRNAs between pregnant and non-pregnant women. The specific hypotheses were: i) prohypertrophic and antifibrotic miRNAs would exhibit increased expression in the third trimester of physiologic pregnancy; ii) antihypertrophic and profibrotic miRNAs would exhibit decreased expression in the third trimester of physiological pregnancy; iii) the $\Delta$ cycle quantification $(\Delta \mathrm{Cq})$ values of prohypertrophic miRNAs would be positively correlated with the thickness of the left ventricle walls; and iv) the $\Delta \mathrm{Cq}$ values of profibrotic miRNAs would be correlated with diastolic dysfunction parameters.

\section{Materials and methods}

Study population and protocol. In the present case-controlled study, conducted between October 2014 and June 2017, healthy women $>18$ years of age with singleton pregnancies and gestational age corresponding to the third trimester were included. The median age of pregnant women was 31 years (range 20-41 years) and of the non-pregnant controls was 28 years (range 21-43 years). Women with a history of cardiovascular diseases or interventions, gestational hypertension, or preeclampsia or eclampsia in the current pregnancy were excluded from the study. The results obtained in the group of pregnant women were compared with those obtained in healthy young non-pregnant female volunteers. Volunteers were enlisted by disseminating information about the study through posters distributed in the Institute of Mother and Child and announcements during classes with nursing students. Both pregnant women and the controls underwent an echocardiographic examination to assess cardiac morphology, valve function, systolic and diastolic function. In the present study, a focus was placed on select echocardiographic variables describing cardiac morphology and function. For cardiac morphology, these were diastolic diameters of intraventricular septum, posterior wall and left ventricle. For systole, these were systolic ejection fraction and maximal blood flow velocity of the aortic valve. Additionally, diastolic function (peak velocity of early diastolic transmitral flow to late transmitral flow ratio, early diastolic velocity of medial and lateral part of mitral annulus) was assessed. The protocol used in the present study was approved by the Local Bioethics Committee at the Institute of Mother and Child, and written informed consent was obtained from all participants. The study was performed in accordance with the guidelines described in the Declaration of Helsinki (13).

Sample preparation and RNA extraction. Upon collection, whole blood samples were centrifuged at $25^{\circ} \mathrm{C}$ for $20 \mathrm{~min}$ at $2,000 \mathrm{x} \mathrm{g}$, and obtained sera were stored at $-80^{\circ} \mathrm{C}$ until further analysis. Total RNA containing a fraction of miRNA was extracted from $400 \mu \mathrm{l}$ of each serum sample using a mirVana PARIS kit (Ambion; Thermo Fisher Scientific, Inc.) according to the manufacturer's protocol.

Determination of miRNA expression. Expression of miRNAs was analyzed using Custom TaqMan Array miRNA Cards (Applied Biosystems; Thermo Fisher Scientific, Inc.). First, selected miRNAs were used to synthesize cDNA using TaqMan MicroRNA Reverse Transcription kit (Applied Biosystems; Thermo Fisher Scientific, Inc.). Briefly, 6 ng isolated RNA in a final volume of $3 \mu \mathrm{l}$ was added to the reverse transcription (RT) reaction mix consisting of $6 \mu 1$ custom RT primer pool, $0.3 \mu \mathrm{l}$ dNTPs with dTTP (100 mM), $3 \mu \mathrm{l}$ MultiScribe Reverse Transcriptase (50 U/ $\mu \mathrm{l}), 1.5 \mu \mathrm{l}$ 10x RT buffer, $0.19 \mu 1$ RNase inhibitor (20 U/ $\mu \mathrm{l}$ ) (Applied Biosystems; Thermo Fisher Scientific, Inc) and $1.01 \mu 1$ nuclease-free water (Ambion; Thermo Fisher Scientific, Inc.). cDNA was synthesized according to the manufacturer's protocol (Applied Biosystems; Thermo Fisher Scientific, Inc). Subsequently, the preamplification reaction was performed using TaqMan PreAmp Master mix and 2X custom PreAmp primer pool (Applied Biosystems; Thermo Fisher Scientific, Inc.), according to the manufacturer's protocol. Briefly, $22.5 \mu \mathrm{l}$ of the reaction mix was added to $2.5 \mu \mathrm{l}$ of each product from the reverse transcription, and then, the reaction was amplified using thermocycling conditions specified in the manufacturer's protocol (Applied Biosystems; Thermo Fisher Scientific, Inc). After the reaction was complete, each PreAmp product was diluted 8-fold with 0.1X TE buffer (pH 8.0) (Ambion; Thermo Fisher Scientific, Inc.) to a final volume of $200 \mu 1$. To prepare the $\mathrm{qPCR}$ reaction mix, $1.13 \mu \mathrm{l}$ diluted PreAmp product was added to the reaction mix consisting of 56.25 $\mu \mathrm{l}$ TaqMan Universal MasterMix II, No AmpErase UNG (2X) (Applied Biosystems; Thermo Fisher Scientific, Inc.) and $55.12 \mu \mathrm{l}$ nuclease-free water (Ambion; Thermo Fisher Scientific, Inc.), and mixed. Subsequently, $100 \mu \mathrm{l}$ of the complete qPCR reaction mix was loaded into a single port on the TaqMan Array miRNA card, centrifuged $\left(2 \times 1 \mathrm{~min}, 331 \mathrm{x} \mathrm{g}\right.$ at $\left.25^{\circ} \mathrm{C}\right)$ and mechanically sealed. Each $\mathrm{qPCR}$ reaction was performed in triplicate using a ViiA7 Real-Time PCR system using thermocycling conditions specified by the manufacturer (Applied Biosystems; Thermo Fisher Scientific, Inc.). The Cq cut-off for the detection was 40 cycles. The results of qPCR were analyzed using automatic baseline and manual threshold set at 0.2 for all samples. U6 small nuclear RNA was used as the endogenous control. Relative expression of analyzed miRNAs was calculated using the $2^{-\Delta \Delta \mathrm{Cq}}$ method (14). The expression 
Table I. Hemodynamic and echocardiographic parameters.

\begin{tabular}{|c|c|c|c|}
\hline Parameter & Pregnancy group ${ }^{a}$ & Control group ${ }^{a}$ & P-value \\
\hline Systolic blood pressure, $\mathrm{mmHg}$ & $115 \pm 10$ & $120 \pm 11$ & 0.16 \\
\hline Diastolic blood pressure, $\mathrm{mmHg}$ & $70.6 \pm 7$ & $74.5 \pm 6$ & $0.049^{\mathrm{b}}$ \\
\hline Heart rate, beats per minute & $87.7 \pm 11.6$ & $76.6 \pm 16$ & $0.0003^{\mathrm{d}}$ \\
\hline Ejection fraction, $\%$ & $62.2 \pm 4.7$ & $65.9 \pm 3.6$ & $0.0015^{\mathrm{c}}$ \\
\hline Left ventricular diameter at end-diastole, $\mathrm{mm}$ & $45.9 \pm 3.6$ & $43.9 \pm 5.1$ & 0.14 \\
\hline Interventricular septum thickness at end-diastole, $\mathrm{mm}$ & $8.7 \pm 1.4$ & $7.9 \pm 1.1$ & $0.028^{\mathrm{b}}$ \\
\hline Left ventricular posterior wall thickness at end-diastole, $\mathrm{mm}$ & $8.6 \pm 1.2$ & $7.7 \pm 0.9$ & $0.005^{\mathrm{c}}$ \\
\hline $\mathrm{E} / \mathrm{A}$ & $1.37 \pm 0.32$ & $1.6 \pm 0.34$ & $0.0084^{\mathrm{c}}$ \\
\hline E/E'med & $5.86 \pm 2.5$ & $6.17 \pm 1.1$ & 0.19 \\
\hline E/E'lat & $5.02 \pm 1.9$ & $4.82 \pm 1.2$ & 0.94 \\
\hline Ao $V \max , \mathrm{m} / \mathrm{s}$ & $1.42 \pm 0.19$ & $1.22 \pm 0.09$ & $<0.0001^{\mathrm{d}}$ \\
\hline
\end{tabular}

${ }^{\mathrm{a}}$ Mean \pm standard deviation. ${ }^{\mathrm{b}} \mathrm{P}<0.05,{ }^{\mathrm{c}} \mathrm{P}<0.01,{ }^{\mathrm{d}} \mathrm{P}<0.001$. E/A, peak velocity of early diastolic transmitral flow to late transmitral flow; E/E'med, peak velocity of early diastolic transmitral flow to peak velocity of early diastolic mitral annular motion at the septal side; E/E'lat, peak velocity of early diastolic transmitral flow to peak velocity of early diastolic mitral annular motion at the lateral side; Ao Vmax, peak aortic velocity.

levels of the following 24 miRNAs were analyzed: miR-1 (assay ID 000385), miR-15b (assay ID 000390), miR-17-5 (assay ID 000393), miR-21 (assay ID 000397), miR-22 (assay ID 002301), miR-26a (assay ID 000404), miR-27b (assay ID 000409), miR-29-a (assay ID 002112), miR-29c (assay ID 000415), miR-30c (assay ID 00419), miR-34a (assay ID 000425), miR-101 (assay ID 002253), miR-124 (assay ID 001182), miR-133a (assay ID 000458), miR-146a (assay ID 000468), miR-191 (assay ID 002299), miR-195 (assay ID 000494), miR-199a-3p (assay ID 002304), miR-199b (assay ID 000500), miR-208a-5p (assay ID 462036_mat), miR-210 (assay ID 000512), miR-222 (Assay ID 000525), miR-328 (assay ID 00543) and miR-1249 (assay ID 002868). Assay ID numbers refer to the catalogue numbers of TaqMan Real-time PCR assays (Thermo Fisher Scientific Inc.).

Analysis of miRNA results. miRNA expression is presented as frequencies, (number of subjects in whom the expression of a given miRNA exceeded the detection limit divided by the sample size) normalized to the U6 $\Delta \mathrm{Cq}$ values, and as the fold change difference in miRNA expression between pregnant and non-pregnant women.

First, the expression of miRNAs was measured as $\mathrm{Cq}$ values. In brief the $\mathrm{Cq}$ value was the number of cycles of amplification of the selected miRNA that allowed for their detection. Then, the $\Delta \mathrm{Cq}$ values were obtained by subtracting U6 from the $\mathrm{Cq}$ value of the analyzed miRNA for each participant. The distributions of U6 in both groups were normal, and the between-group differences in U6 expression did not differ significantly (mean 31.94 for non-pregnant controls vs. 31.13 for pregnant women, $\mathrm{P}=0.09$ ). Additionally, in a subgroup of patients, an external spike, cel-39 (exogenous control molecule), was used. The median value of cel-39 in the pregnancy group was 20.26 \pm 2.06 (range 18.7-33.5) and for the control group, 20.4 \pm 1.6 (range 19.6-24.5).

$\Delta \mathrm{Cq}$ should be interpreted as a marker of expression levels. The expression of each miRNA is inversely proportional to its $\Delta \mathrm{Cq}$ value. The lower the $\Delta \mathrm{Cq}$ values, the higher the expression levels of a given miRNA. Conversely, larger $\Delta \mathrm{Cq}$ values should be interpreted as lower expression levels of miRNAs.

Whenever the $\Delta \mathrm{Cq}$ value for a given miRNA differed significantly between the study groups, the fold change was calculated. First the $\Delta \Delta \mathrm{Cq}$ value was obtained by subtracting the control groups median $\Delta \mathrm{Cq}$ value from the pregnancy groups median $\Delta \mathrm{Cq}$ value of the analyzed miRNA. Then, fold change was calculated using $2^{-\Delta \Delta C q}$ formula (14). The fold change is a measure of the difference in the expression levels of a given compound between two groups.

Statistical analysis. As the distributions of most $\mathrm{Cq}$ values was skewed, non-parametric tests were used for statistical analysis. Statistical characteristics of continuous variables are presented as medians and ranges/interquartile ranges (IQR), except for the hemodynamic and echocardiographic parameters, which are presented as the mean \pm standard deviation, as they were normally distributed across both groups. Distributions of categorical variables are presented as numbers (percentages). Statistical significances of between-group comparisons in the non-parametric variables were verified using a Mann-Whitney U-test. Distributions of nominal variables were compared using a $\chi^{2}$ test. Relationships between the expression levels of the selected miRNAs and echocardiographic parameters were analyzed using Spearman's rank correlation coefficient analysis. All statistical calculations were performed using theSAS/STAT version 14.3 (SAS Institute, Inc.). $\mathrm{P}<0.05$ was considered to indicate a statistically significant difference.

Characteristics of the selected miRNAs. The analyzed cardiac miRNAs were categorized into three groups. The first group consisted of miRNAs associated with cardiac hypertrophy (miR-1, miR-17-5, miR-22, miR-34a, miR-124, miR-133a, miR-195, miR-199a-3p, miR-199b, miR-210, miR-222 and miR-1249). The second consisted of miRNAs associated with cardiac hypertrophy and fibrosis (miR-15b, miR-21, miR-26a, miR-29-a, miR-29c, miR-30c, miR-101, miR-146a, miR-191, 
Table II. Median $\Delta \mathrm{Cq}$ values for cardiac miRNAs expressed in more than half of the study participants during the third trimester.

\begin{tabular}{|c|c|c|c|c|}
\hline Group & miRNA & Median $\Delta \mathrm{Cq}$ values & Interquartile range & Range \\
\hline \multirow[t]{3}{*}{ Hypertrophy } & miR-17-5 & 1.68 & $0.73-2.82$ & $-1.2-6.4$ \\
\hline & miR-199a & 0.736 & $0.04-1.74$ & $-2.5-5.5$ \\
\hline & miR-222 & -0.17 & -2.79 & $-4.4-4.0$ \\
\hline \multirow[t]{10}{*}{ Hypertrophy and fibrosis } & $\operatorname{miR}-21$ & 8.6 & 7.73-9.45 & 4.6-12.7 \\
\hline & miR-26a & -2.17 & -1.89 & $-6.3-2.9$ \\
\hline & miR-30c & 0.2 & -1.89 & $-3.6-3.8$ \\
\hline & miR-146a & -2.51 & -1.66 & $-5.4-0.9$ \\
\hline & miR-191 & -2.26 & -1.84 & $-5.7-1.6$ \\
\hline & $\operatorname{miR}-29 a$ & 1.49 & $0.4-2.44$ & $-2.2-5.3$ \\
\hline & $\operatorname{miR}-29 c$ & 0.53 & -3.05 & $-2.5-8.5$ \\
\hline & miR-101 & 4.59 & $2.7-7.75$ & $-2.0-10.8$ \\
\hline & $\operatorname{miR}-15 b$ & 1.92 & $1.15-2.75$ & $-1.6-8.2$ \\
\hline & miR-328 & 1.58 & $0.87-3.17$ & $-2.8-8.5$ \\
\hline Systolic function & $\operatorname{miR}-27$ & 3.09 & $2.0-6.35$ & $-0.5-12.5$ \\
\hline
\end{tabular}

Cq, cycle quantification.

miR-208a-5p and miR-328). The third group included only one miRNA, miR-27b, a marker of proper systolic function.

First, the expression levels of the selected cardiac miRNAs were analyzed as dichotomous variables (present vs. absent) and quantified based on the $\Delta \mathrm{Cq}$ values. Then, the between-group differences in the expression levels of the selected miRNAs in pregnant women and non-pregnant controls were determined, with the fold-change values calculated whenever the between-group difference turned out to be statistically significant.

\section{Results}

Group characteristics. The results obtained in a group of 61 pregnant women were compared with those of 19 non-pregnant controls.

The median age of pregnant women was significantly higher compared with non-pregnant controls [31 years (IQR 27-35) vs. 28 years (IQR 22-32), $\mathrm{P}=0.02]$. The study groups did not differ significantly in terms of their (pre-pregnancy) body mass index values $\left(22.04 \mathrm{~kg} / \mathrm{m}^{2}\right.$ in pregnant women vs. $22.48 \mathrm{~kg} / \mathrm{m}^{2}$ in the controls, $\left.\mathrm{P}=0.77\right)$. A total of $36(59 \%)$ women from the pregnant group were primigravidas. Mean gestational age at the time of the study was $32.8 \pm 2.6$ weeks. No significant between-group differences were found in terms of the number of past pregnancies $(\mathrm{P}=0.8)$ (Table $\mathrm{I})$.

The study groups differed with regards to a few hemodynamic and echocardiographic indices (Table I). A few study participants presented with cardiovascular risk factors and comorbidities. The list of concomitant diseases present in the controls included diabetes mellitus $(n=1)$, hypercholesterolemia $(n=1)$, hypothyroidism $(n=1)$ and polycystic ovary syndrome $(n=1)$. In turn, the group of pregnant women included five women with gestational diabetes mellitus without a past history of diabetes, four with hypercholesterolemia, five with hypothyroidism and another five with asthma. The study groups did not differ
Table III. Differences in the expression frequencies of selected microRNAs between healthy pregnant women and nonpregnant controls.

\begin{tabular}{|c|c|c|c|}
\hline $\operatorname{miR}$ & $\begin{array}{c}\text { Pregnant } \\
\text { women, n (\%) }\end{array}$ & $\begin{array}{c}\text { Control } \\
\text { group, n }(\%)\end{array}$ & P-value \\
\hline miR-1 & 1/61(1.6) & $1 / 19(5.3)$ & 0.4 \\
\hline miR-17-5 & $58 / 61(95.1)$ & $18 / 19(94.7)$ & 1.0 \\
\hline miR-22 & $33 / 61(54.1)$ & $11 / 19(57.9)$ & 0.8 \\
\hline miR-34a & 0/61 (0) & 1/19 (5.3) & 0.2 \\
\hline miR-124a & 11/61 (18) & $6 / 19$ (31.6) & 0.2 \\
\hline miR-133a & 17/61 (27.9) & $5 / 19(26.3)$ & 0.9 \\
\hline miR-195 & 40/61 (65.6) & $14 / 19(73.7)$ & 0.5 \\
\hline miR-199a-3p & $60 / 61(98.4)$ & $18 / 19(94.7)$ & 0.42 \\
\hline miR-199b & 2/61 (3.3) & $0 / 19(0)$ & 1.0 \\
\hline miR-210 & 22/61 (36) & $6 / 19(31.6)$ & 0.7 \\
\hline miR-222 & $58 / 61(95.1)$ & 19/19 (100) & 1.0 \\
\hline miR-1249 & $17 / 61(27.9)$ & 7/19 (36.8) & 0.5 \\
\hline miR-15b & $58 / 61(95.1)$ & $16 / 19(84.2)$ & 0.1 \\
\hline miR-21 & 61/61 (100) & 19/19(100) & 1.0 \\
\hline miR-26a & 61/61 (100) & 19/19 (100) & 1.0 \\
\hline miR-29a & $57 / 61(93.4)$ & 15/19 (79) & 0.1 \\
\hline miR-29c & 60/61 (98.4) & $17 / 19(89.5)$ & 0.1 \\
\hline miR-30c & 61/61 (100) & 19/19 (100) & 1.0 \\
\hline miR-101 & 35/61 (57.4) & $10 / 19(52.6)$ & 0.7 \\
\hline miR-146a & 61/61 (100) & 19/19 (100) & 1.0 \\
\hline miR-191 & 61/61 (100) & 19/19 (100) & 1.0 \\
\hline miR-208a-5p & 0/61 (0) & $0 / 19(0)$ & 1.0 \\
\hline miR-328 & $58 / 61(95.1)$ & $18 / 19(94.7)$ & 1.0 \\
\hline $\operatorname{miR}-27 b$ & $41 / 61(67.2)$ & 9/19 (47.4) & 0.1 \\
\hline
\end{tabular}

significantly in the frequency of physical activity $(\mathrm{P}=0.61)$. The proportions of smokers in both groups were low and did 
Table IV. Differences in the expression levels of selected miRNAs between healthy pregnant women and non-pregnant controls.

\begin{tabular}{|c|c|c|c|c|c|}
\hline \multirow[b]{2}{*}{ miRNA } & \multicolumn{2}{|c|}{ Pregnant women } & \multicolumn{2}{|c|}{ Control group } & \multirow[b]{2}{*}{ P-value } \\
\hline & Median $\Delta \mathrm{Cq}(\mathrm{IQR})$ & Range of $\Delta \mathrm{Cq}$ values & Median $\Delta \mathrm{Cq}(\mathrm{IQR})$ & Range of $\Delta \mathrm{Cq}$ values & \\
\hline $\operatorname{miR}-17-5$ & $1.68(0.8-3.0)$ & $(-1.68)-4.96$ & $0.56(-0.3-2.2)$ & $-1.18-6.35$ & 0.056 \\
\hline $\operatorname{miR}-22$ & $6.31(4.8-8.3)$ & $3.18-10.85$ & $4.53(3.2-7.7)$ & $1.62-9.28$ & 0.08 \\
\hline miR-195 & $3.79(2.2-7.2)$ & $(-2.32)-10.15$ & $2.3(1.4-3.5)$ & $-0.08-9.17$ & $0.046^{\mathrm{a}}$ \\
\hline miR-199a-3p & $0.74(0.05-1.8)$ & $(-2.54)-7.96$ & $0.33(-1.4-1.3)$ & $-3.78-4.12$ & 0.26 \\
\hline miR-210 & $7.7(5.9-9.0)$ & $1.34-12.38$ & $7.27(3.9-8.2)$ & $-0.28-5.92$ & 0.96 \\
\hline miR-222 & $-0.17(-1.2-1.6)$ & $(-4.4)-8.19$ & $-1.58[-3.3-(-0.5)]$ & $-5.2-2.9$ & $0.0038^{\mathrm{b}}$ \\
\hline miR-1249 & $8.45(7.7-9.3)$ & $3.54-12.06$ & $7.66(6.3-8.3)$ & $3.2-9.3$ & $0.02^{\mathrm{a}}$ \\
\hline $\operatorname{miR}-15 b$ & $1.92(1.2-2.8)$ & $(-1.6)-8.2$ & $1.14(-0.3-3.4)$ & $-1.83-7.66$ & 0.44 \\
\hline miR-21 & $8.6(7.7-9.4)$ & $4.63-12.7$ & $7.66(6.4-8.3)$ & $2.06-9.28$ & $0.0095^{\mathrm{b}}$ \\
\hline miR-26a & $-2.17[(-3.1-(-1.2)]$ & $(-6.26)-2.9$ & $-3.09[-4.2-(-2.0)]$ & $-6.1-2.65$ & 0.069 \\
\hline $\operatorname{miR}-29 a$ & $1.49(0.4-2.4)$ & $(-2.24)-9.83$ & $1.74(0.8-3.1)$ & $-1.42-7.7$ & 0.37 \\
\hline $\operatorname{miR}-29 c$ & $0.53(-0.4-2.7)$ & $(-2.53)-8.51$ & $0.68(-1.3-6.3)$ & $-2.75-9.3$ & 0.62 \\
\hline $\operatorname{miR}-30 \mathrm{c}$ & $0.2(-0.6-1.2)$ & $(-3.56)-3.8$ & $-0.68[-2.4-(-0.3)]$ & $-3.7-1.23$ & $0.02^{\mathrm{a}}$ \\
\hline miR-101 & $4.6(2.8-7.9)$ & $(-2.05)-10.83$ & $4.37(1.9-7.6)$ & $0.25-9.17$ & 0.49 \\
\hline miR-146a & $-2.51[-3.4-(-1.6)]$ & $(-5.35)-0.89$ & $-3.45[-4.7-(-2.7)]$ & $-5.96-(-1.07)$ & $0.01^{\mathrm{a}}$ \\
\hline miR-191 & $-2.26[-3.3-(-1.5)]$ & $(-5.7)-1.63$ & $-2.82[-4.8-(-2.3)]$ & $-6.13-(-1.37)$ & 0.052 \\
\hline $\operatorname{miR}-328$ & $1.58(0.9-3.2)$ & $(-2.79)-8.46$ & $0.85(-0.3-1.5)$ & $-2.23-3.63$ & $0.01^{\mathrm{a}}$ \\
\hline $\operatorname{miR}-27 b$ & $3.1(2.0-6.4)$ & $(-0.49)-12.51$ & $4.36(0.9-7.9)$ & $-0.65-11.33$ & 1 \\
\hline
\end{tabular}

${ }^{\mathrm{a}} \mathrm{P}<0.05,{ }^{\mathrm{b}} \mathrm{P}<0.01 . \mathrm{miR} / \mathrm{miRNA}$, microRNA; $\mathrm{Cq}, \mathrm{Cycle}$ quantification; IQR, interquartile range.

not differ significantly $(3.5 \%$ in pregnant women vs. $5.3 \%$ in the controls; $\mathrm{P}=0.72)$.

Expression of cardiac miRNAs in the third trimester. Amongst the miRNAs associated with cardiac hypertrophy, pregnant women expressed miR-17-5 (58/61, 95\%), miR-22 (22/50, 44\%), miR133a (17/61, 28\%), miR-195 (40/61, 66\%), miR-199a (60/61, 98\%), miR-199b (2/61, 3\%), miR-210 (22/61, $36 \%)$, miR-222 (58/61, 95\%) and miR-1249 (17/61, 28\%). The expression of miR-1 was found in only one pregnant woman (1.67\%), and none of the pregnant women expressed miR-34a and miR-124.

All pregnant women expressed miRNAs associated with cardiac hypertrophy and fibrosis (miR-21, miR-26a, miR-30c, miR-146a and miR-191). Additionally, the other miRNAs from this group, miR-15b (58/61, 95\%), mir-29a (57/61, 93\%), miR-29c $(60 / 61,98 \%)$, miR-101 (40/61, 66\%) and miR-328 $(58 / 61,95 \%)$, were found to be expressed in a considerable proportion of pregnant women. In contrast, none of the study participants expressed miR-208a.

Expression of the only miRNA from the third group, miR-27b, was observed in 41 out of $61(67 \%)$ pregnant women.

Median $\triangle C q$ values for the selected cardiac miRNAs in the third trimester. Median $\Delta \mathrm{Cq}$ values for all miRNAs, the expression of which was observed in $>50 \%$ of the study participants, are shown in Table II.

Comparison with the control group. Pregnant women and non-pregnant controls did not differ significantly in terms of the expression frequencies of all analyzed miRNAs (Table III). However, several statistically significant between-group differences were found with regards to $\Delta \mathrm{Cq}$ values, suggesting that pregnant women differed from non-pregnant controls in terms of the expression levels of certain miRNAs (Table IV). The between-group comparisons of the $\Delta \mathrm{Cq}$ values were performed solely for the miRNAs that were expressed in $>30 \%$ of the study subjects. The expression levels of miRNAs which were significant differentially expressed between pregnant and non-pregnant women or at least a trend towards differential expression $(\mathrm{P}<0.1)$ was found, stratified according to their function, are presented in Figs. 1 and 2.

Fold-change. Among miRNAs with statistically significant differences in the expression levels in healthy pregnant women and non-pregnant controls, all the miRNAs, regardless of their function, exhibited lower expression levels in pregnant women. The fold-changes in the expression levels of these miRNAs in pregnant women are shown in Fig. 3.

Correlations between select miRNAs and echocardiographic parameters. Correlations with echocardiographic indices were analyzed only if at least a trend towards a significant difference in $\Delta \mathrm{Cq}$ values for a given miRNA were observed between pregnant women and non-pregnant controls $(\mathrm{P}<0.1)$. These results are presented in Table V).

Morphology. Significant correlations and noticeable trends were found between interventricular septum thickness and left ventricular posterior wall thickness at end-diastole and 

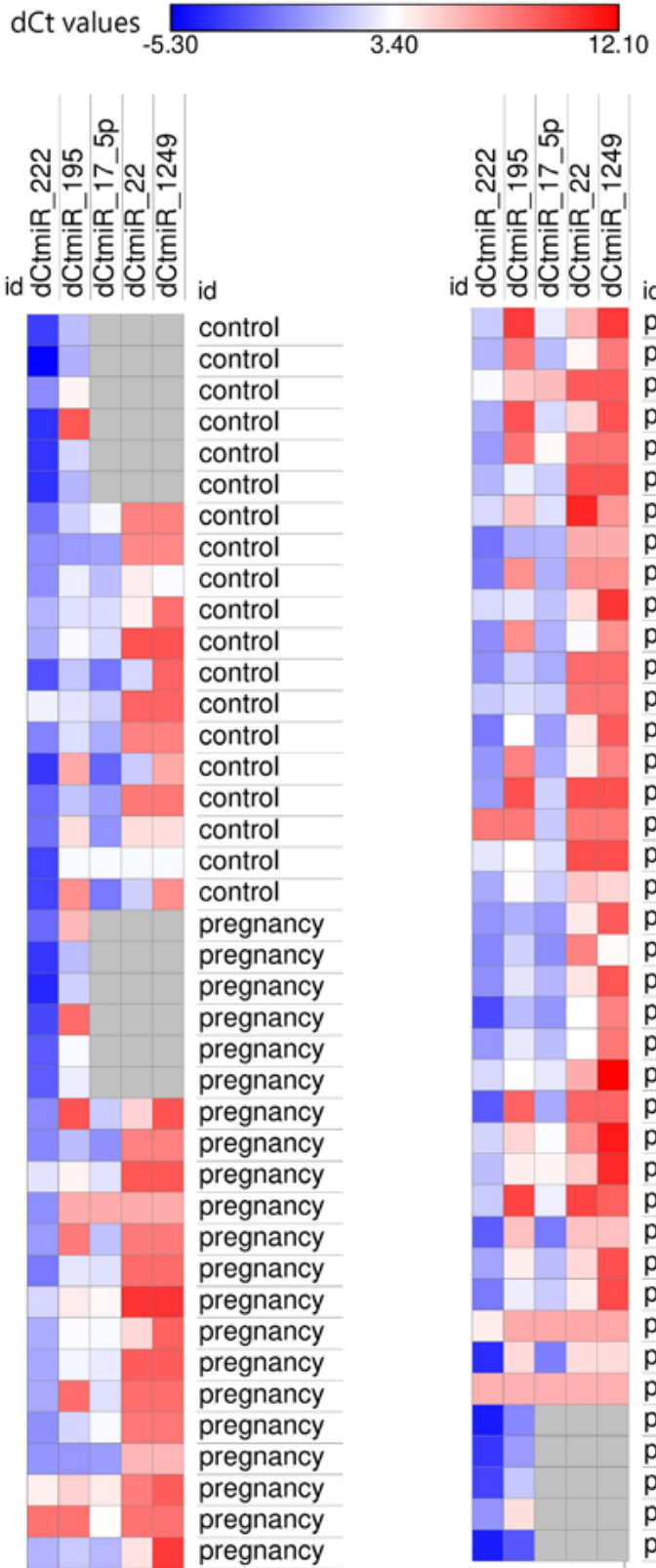

Figure 1. Heatmap of the expression levels of microRNAs associated with hypertrophy in pregnant subjects and controls. Navy blue, high expression levels (low $\Delta$ Cq values); red, low expression levels (high $\Delta C q$ values). dcmiR; $\mathrm{Cq}$, Cycle quantification.

the expression levels of certain miRNAs. The intraventricular septum thickness at end-diastole was inversely correlated with the $\Delta \mathrm{Cq}$ value of miR-21. Furthermore, inversely correlated trends were found between echocardiographic parameters and $\Delta \mathrm{Cq}$ values for miR-1249 (correlation with intraventricular septum thickness) and miR-30c (correlation with intraventricular septum thickness and peak velocity of early diastolic transmitral flow to peak velocity of early diastolic mitral annular motion at the septal side). In turn, the left ventricular posterior wall thickness at end-diastole correlated positively with $\Delta$ Cq for miR-222 (Table V).

Systolic function. $\Delta \mathrm{Cq}$ for miR-195 was positively correlated with left ventricular ejection fraction and showed an inverse correlation with peak aortic velocity (Table V).

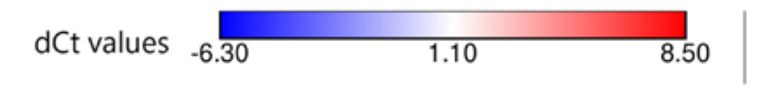

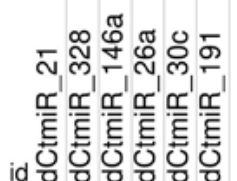

d

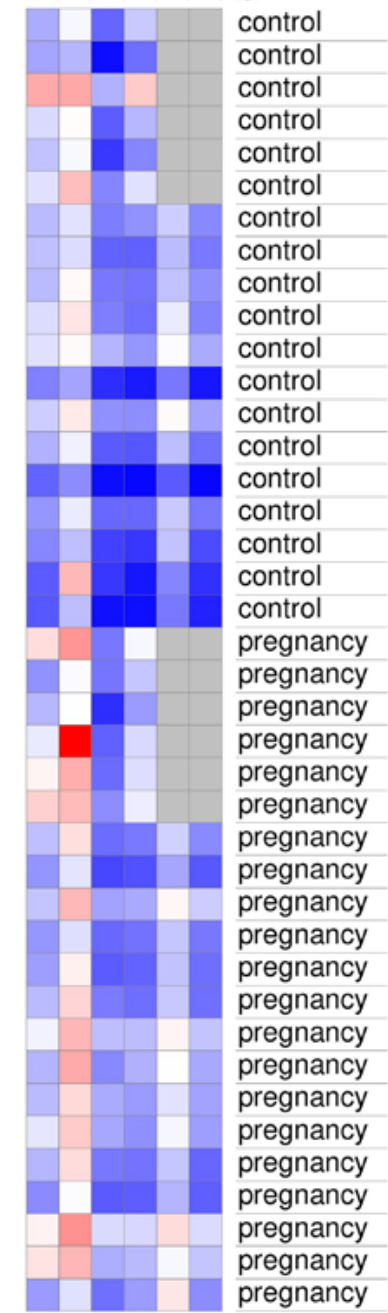

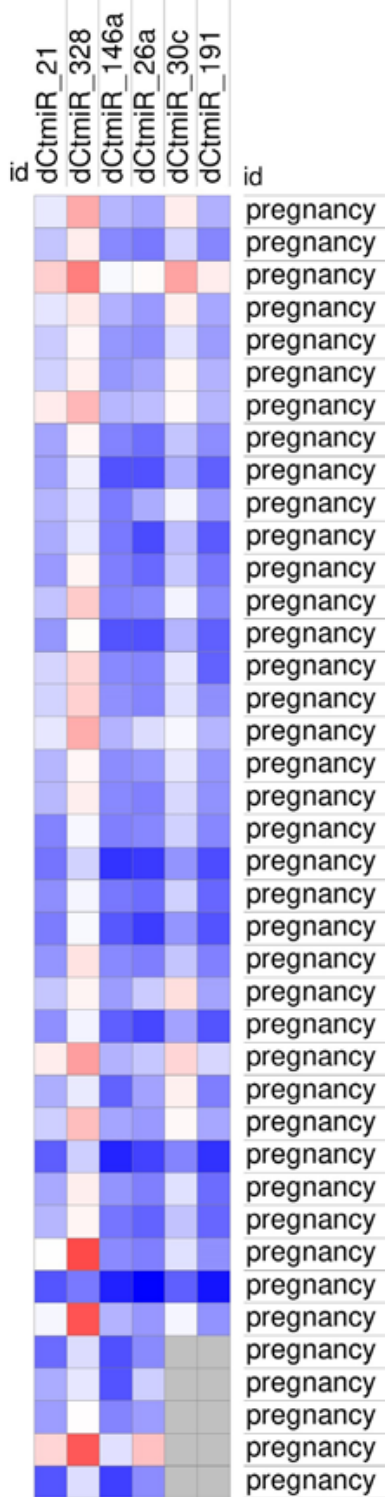

Figure 2. Heatmap of the expression levels of microRNAs associated with hypertrophy and fibrosis in pregnant subjects and controls. Navy blue, high expression levels (low $\Delta \mathrm{Cq}$ values); red, low expression levels (high $\Delta \mathrm{Cq}$ values). dcmiR; Cq, Cycle quantification.

Diastolic function. A number of significant correlations were found between the expression levels of various miRNAs and parameters of diastolic function. Interestingly, except for miR-17-5, miRNAs which were correlated with parameters describing mitral annular motion at the septal side did not show significant associations with the parameters of mitral annular motion at the lateral side and vice versa (Table V).

Correlations in non-pregnant controls. In non-pregnant controls, the expression levels of analyzed miRNAs did not correlate significantly with either ejection fraction or peak aortic velocity, and the profiles of correlations with the left ventricular posterior wall thickness, interventricular septum thickness and parameters of diastolic function differed compared with the pregnant women. Regarding cardiac 


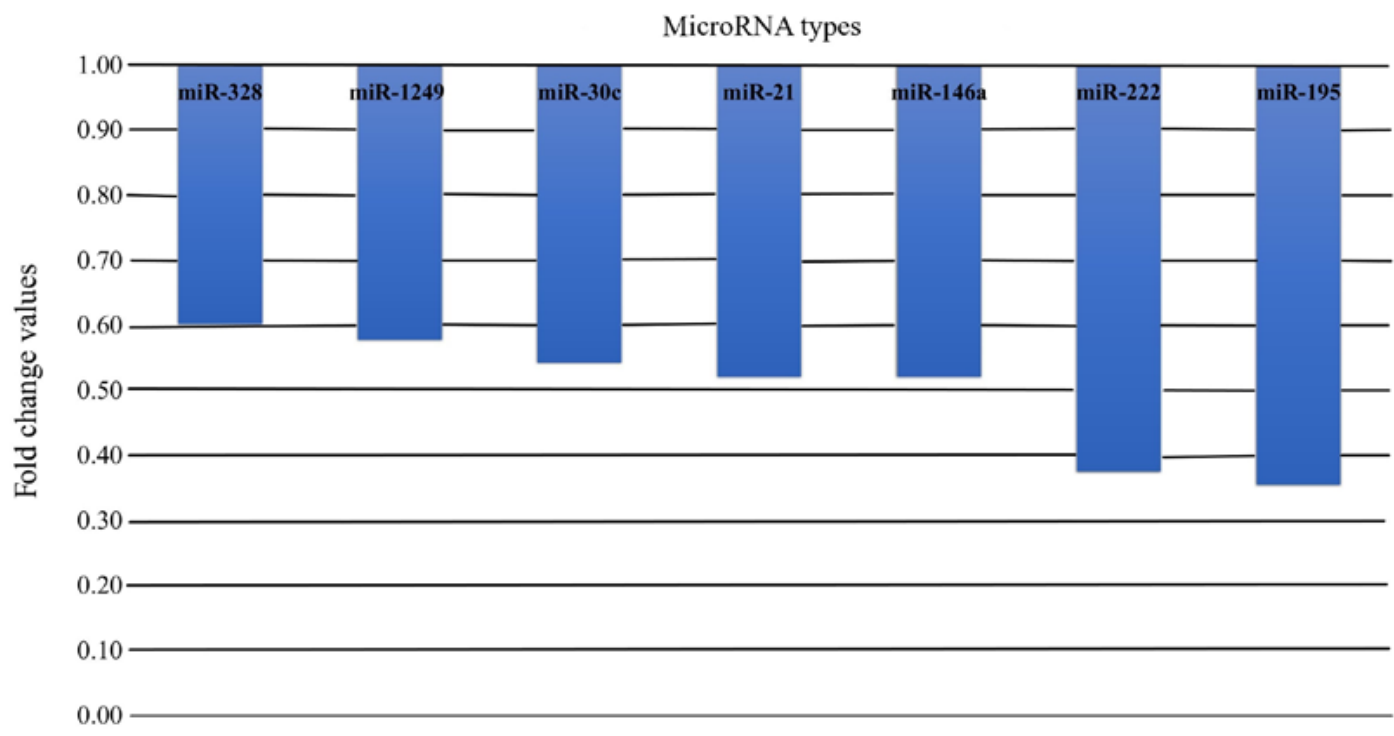

Figure 3. MicroRNAs with a significant fold change between pregnant and non-pregnant women. miR, microRNA.

morphology, a negative correlation was observed between posterior wall thickness and miR-222 ( $\mathrm{r}=-0.8), \mathrm{miR}-191$ $(\mathrm{r}=-0.6)$ and a positive correlation was observed between intraventricular wall thickness and miR-22 ( $\mathrm{r}=0.7)$, miR-17-5 $(\mathrm{r}=0.6)$, miR-191 $(\mathrm{r}=0.6)$, miR-30c $(\mathrm{r}=0.6)$. In the aspect of diastolic function a negative correlation was observed between peak velocity of early diastolic transmitral flow to late transmitral flow ratio and miR-29c $(r=-0.5)$. miR-29c was negatively correlated with peak velocity of early diastolic mitral annular motion at the septal side $(\mathrm{r}=-0.6)$. There were no correlations between miR concentration and analyzed systolic function parameters (data not shown).

\section{Discussion}

Relatively little is known regarding the role of cardiovascular miRNAs in physiological myocardial hypertrophy during pregnancy. In the present study, a detailed analysis of cardiovascular miRNAs expressed during the third trimester of pregnancy was performed, when pregnancy-associated volume overload is at its highest.

During the design of the present study a difference in cardiac miRNA expression between pregnant and non-pregnant women was hypothesized. Although there were no differences in the specific miRNAs expressed, the expression levels of miR-195, miR-222, miR-1249, miR-21, miR-30c, miR-146a and miR-328 were lower in pregnant women. The similarities in the qualitative composition of the assessed miRNAs suggested that the mechanisms controlling cardiomyocytes and extracellular matrix composition in these two groups may be essentially the same; however, the differences in the miRNA expression levels highlight their likely influence on pregnancy related remodeling. Importantly, despite a volume overload typical for physiological pregnancy, the expression levels of miRNAs associated with myocardial damage, such as miR-208, were not altered.

Neither the prohypertrophic, nor the antifibrotic microRNAs exhibited increased expression in the third trimester of physiological pregnancy. Among the prohypertrophic miRNAs, miR-30c (15), miR-195 (16) and miR-328 (15) were downregulated compared with the healthy controls. Interestingly, miR-222, an antifibrotic miRNA which is inversely correlated with the degree of myocardial fibrosis through inhibition of transforming growth factor- $\beta$ (17), was downregulated during pregnancy. The decrease in miR-328 expression in pregnancy, first reported in our previous study (18), has now been confirmed in a larger cohort.

A hypothesis of the present study was that expression of antihypertrophic and profibrotic miRNAs would be decreased in the third trimester of physiological pregnancy. However, expression of the studied antihypertrophic miRNAs were similar in both groups. Among the profibrotic miRNAs, a significant difference was observed in expression of miR-21. In myocardial infarction models, miR-21 stimulated collagen synthesis $(19,20)$. Thus, it is hypothesized that the decrease in miR-21 expression may protect the myocardium of pregnant women against fibrosis resulting from volume overload in pregnancy.

Additionally a decrease in miR-146a expression was observed, and this miRNA possesses both an antimetabolic function, suppressing mitochondrial metabolism (21) and a profibrotic role (22). The decreased expression observed in pregnant women may be an adaptive mechanism, allowing cardiomyocytes to increase mitochondrial metabolism. Furthermore, miR-146a is the only miRNA associated with peripartum cardiomyopathy (21). In this disease, miR-146a released form endothelial cells following stimulation with $16 \mathrm{kDa} \mathrm{N}$-terminal fragments of prolactin is absorbed by cardiomyocytes, inhibiting their metabolic activity (21). This mechanism may also underlie the decrease in myocardial contractility observed in peripartum cardiomyopathy (10).

To analyze potential associations between miRNAs and echocardiographic indices, a series of statistical analyses was performed. Echocardiographic data collected during the present study were consistent with published evidence; pregnant women in the third trimester possessed thicker cardiac walls, higher peak aortic velocity, lower left ventricular ejection fraction and altered parameters of diastolic function. 


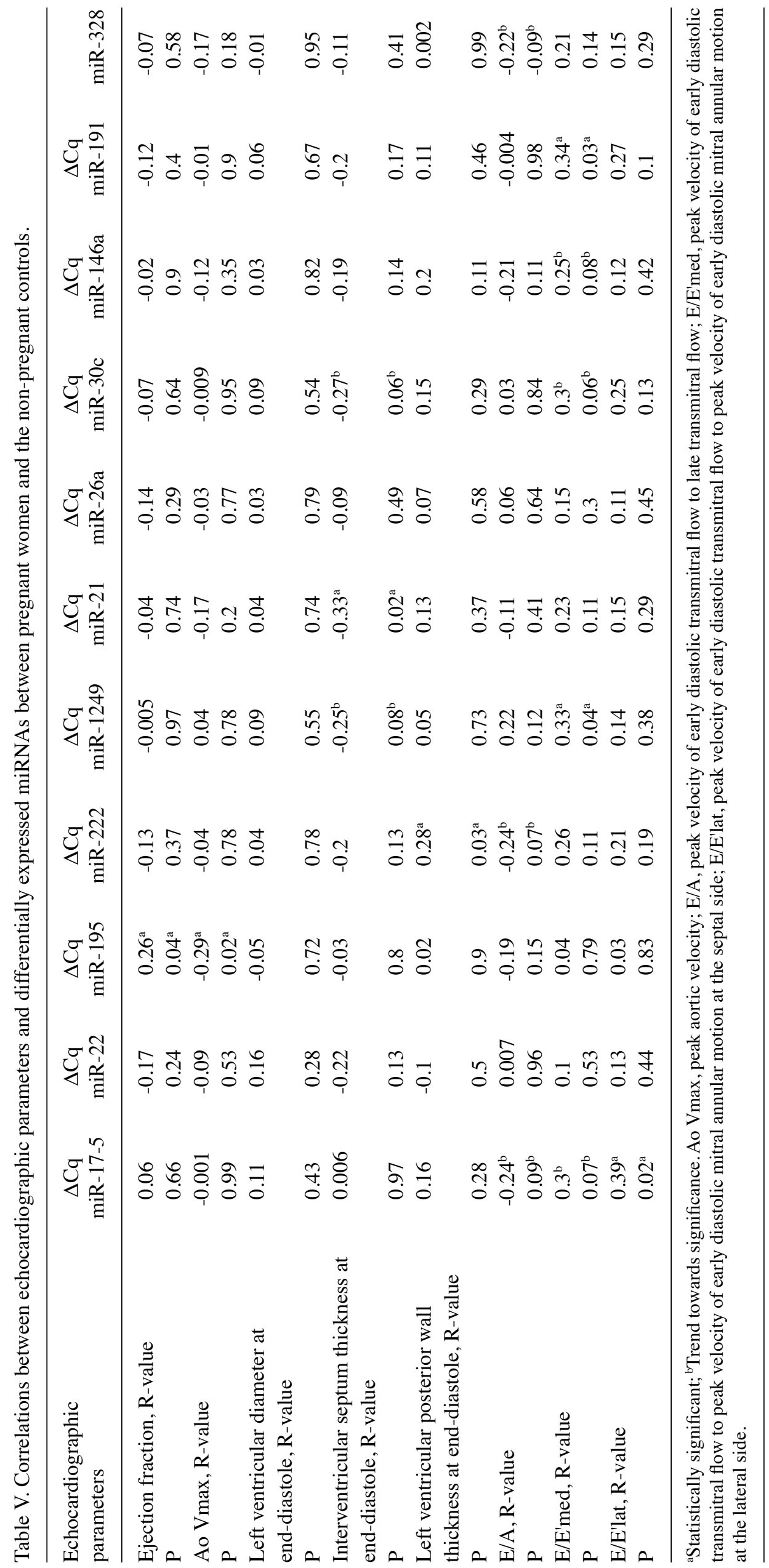


These changes seem to be consequences of volume overload in pregnancy that secures a fetus' demand for nutrients delivered via maternal circulation.

The differences between correlations observed in pregnant women and non-pregnant controls suggest that the analyzed miRNAs may serve a role in myocardial remodeling during pregnancy, and their function in pregnant women may differ than during physiological conditions. A correlation between $\Delta \mathrm{Cq}$ values of prohypertrophic miRNAs and left ventricle wall thickness was hypothesized. Additionally, a dependence between profibrotic miRNAs and diastolic left ventricle function parameters was also hypothesized. The results suggested that hypertrophy of the interventricular septum and free cardiac wall (represented by posterior wall thickness) in pregnancy was achieved via different underlying mechanisms. Interventricular septum thickness was positively correlated with the expression of miR-21, whereas an inverse correlation was found between the thickness of the posterior wall and miR-222 expression. This observation was surprising as miR-222 is considered a vital miRNA required for proliferation and growth of cardiomyocytes in response to physical exercise $(23,24)$. In human and animal exercise models miR-222 increases myosin heavy chain $\alpha / \beta$ ratio. In animal models administration of miR-222 antagonist resulted in complete abrogation of heart size enlargement in response to intensive exercise (24). Based on the results of the present and the aforementioned previous studies, adaption mechanisms to exercise and pregnancy overload may have different molecular basis, and the differences may be due the alternative models of overload-in pregnancy the volume overload is constant and stably increasing over several weeks, whereas during exercise, overload appears in intervals and the increase is more varied and short-lasting.

Analysis of miRNA expression and hemodynamic parameters revealed an inverse correlation between the expression of miR-195 and left ventricular ejection fraction, along with a positive correlation between miR-195 expression levels and peak aortic velocity. In contrast, none of the miRNAs were associated with the assessed hemodynamic indices in non-pregnant women.

The correlations between the diastolic parameters and expression of miR-17-5p seem to be of particular import. Higher expression levels of miR-17-5p were observed to be associated with improved relaxation, as shown by higher values of the $\mathrm{E}$ to $\mathrm{A}$ wave ratio and lower estimated left ventricular filling pressures, shown by lower values of the $\mathrm{E}$ to E'med and $\mathrm{E}$ to E'lat ratios (see Table $\mathrm{V}$ for detailed results). Experimental mouse models of myocardial infarction demonstrated that knockdown of miR-17-5p expression decreased infarction area and the area of fibrosis and stimulated endothelial growth (25). Therefore, decreased expression of miR-17-5p may serve a regulatory role in diastolic function adaptation in pregnancy. Furthermore, there was a tendency towards positive correlations between left ventricular myocardial relaxation (E/A ratio) and the expression levels of miR-222 and miR-328.

The present study has some limitations. Age differences between studied groups needs to be taken into account when interpreting the results. Age is a factor that can affect miRNA expression levels. Although, the difference in age between the groups was statistically significant, the numerical difference was only 3 years. Available data suggests that the effect of age on miRNA expression levels requires decades (26). Furthermore, there were no correlations between expression of any of the miRNAs assessed with age in any of the groups. Therefore, it is unlikely that age had a significant impact on the observed differences in miRNA expression levels. Another limitation was that the present study was a case control study. Any differences in demographics could not be avoided due to the design of the study.

The relatively low expression levels of the studied miRNAs with a significant number of obtained $\mathrm{Cq}$ values $>30$ constitutes a limitation as well. This may have been a result of low RNA input. In order to ensure the highest quality of results, the RNA input was unified across all participants. The lowest quantity of RNA obtained from the samples from all 80 participants was $6 \mathrm{ng}$. Median Cq values of U6 (internal normalization) and studied miRNA compared with median $\mathrm{Cq}$ values of cel-39 (external normalization) highlights the fact that there the absolute expression levels of the studied miRNAs was very low in both groups, and not the result of a low RNA input.

In conclusion, while the profiles of cardiac miRNAs expressed in healthy pregnant women and healthy nonpregnant controls were similar, these two groups differed in terms of miRNA expression levels of select miRNAs. In the third trimester of physiological pregnancy, downregulation of miR-17-5p, miR-21, miR-30c, miR-146a, miR-195, miR-222 and miR-328 expression was observed. The differences in the correlations of echocardiographic indices with miRNAs in pregnant and non-pregnant women suggests that miRNAs may regulate both the structure and function of the heart in pregnant women, influencing cardiac muscle thickness, as well as systolic and diastolic function.

\section{Acknowledgements}

We would like to thank Professor Katarzyna Szamotulska, Dr Leszek Soluch and Dr Katarzyna Markiewicz (Institute of Mother and Child) for their assistance.

\section{Funding}

The present study was supported by a grant from the Polish National Center of Science (grant no. NCN 2013/11/N/NZ5/03388).

\section{Availability of data and materials}

The datasets used and/or analyzed during the current study are available from the corresponding author on reasonable request.

\section{Authors' contributions}

All authors contributed to the conception and design of the study and writing of the manuscript. ES, AB, KP, GS and AF recruited the participants, obtained informed consent and were responsible for the collection blood samples. AZ and MM performed the microRNA assays. ES assisted during microRNA measurements. ES managed the database. ES, 
AZ, GO, TM, MM and AF interpreted the results of statistical analysis. All authors read and approved the final manuscript.

\section{Ethics approval and consent to participate}

The protocol used in the present study was approved by the Local Bioethics Committee at the Institute of Mother and Child, and written informed consent was obtained from all participants.

\section{Patient consent for publication}

Not applicable.

\section{Competing interests}

The authors declare that they have no competing interests.

\section{References}

1. Sanghavi M and Rutherford JD: Cardiovascular physiology of pregnancy. Circulation 130: 1003-1008, 2014.

2. Indira Devi C and Srikanth S: Cardiovascular changes during pregnancy, labour and puerperium. Int J Sci Res 4: 555-561, 2015.

3. Umar S, Nadadur R, Iorga A, Amjedi M, Matori H and Eghbali M: Cardiac structural and hemodynamic changes associated with physiological heart hypertrophy of pregnancy are reversed postpartum. J Appl Physiol 113: 1253-1259, 2012.

4. Fok WY, Chan LY, Wong JT, Yu CM and Lau TK: Left ventricular diastolic function during normal pregnancy: Assessment by spectral tissue Doppler imaging. Ultrasound Obstet Gynecol 28: 789-793, 2006.

5. Li J, Umar S, Amjedi M, Iorga A, Sharma S, Nadadur RD, Regitz-Zagrosek V and Eghbali M: New frontiers in heart hypertrophy during pregnancy. Am J Cardiovasc Dis 2: 192-207, 2012.

6. Condorelli G, Latronico MVG and Cavarretta E: microRNAs in cardiovascular diseases: Current knowledge and the road ahead. J Am Coll Cardiol 63: 2177-2187, 2014.

7. Silva GJJ, Bye A, El Azzouzi H and Wisløff U: MicroRNAs as important regulators of exercise adaptation. Prog Cardiovasc Dis 60: 130-151, 2017.

8. Mohseni Z, Spaanderman MEA, Oben J, Calore M, Derksen E, Al-Nasiry S, de Windt LJ and Ghossein-Doha C: Cardiac remodelling and pre-eclampsia: An overview of overlapping miRNAs. Ultrasound Obstet Gynecol 52: 310-317, 2018.

9. Li J, Wu G, Cao Y and Hou Z: Roles of miR-210 in the pathogenesis of pre-eclampsia. Arch Med Sci 15: 183-190, 2019.

10. Halkein J, Tabruyn SP, Ricke-Hoch M, Haghikia A, Nguyen NQ, Scherr M, Castermans K, Malvaux L, Lambert V, Thiry M, et al: MicroRNA-146a is a therapeutic target and biomarker for peripartum cardiomyopathy. J Clin Invest 123: 2143-2154, 2013.

11. Yang Y, Rodriguez JE and Kitsis RN: A microRNA links prolactin to peripartum cardiomyopathy. J Clin Invest 123: 1925-1927, 2013.

12. O'Brien J, Hayder H, Zayed Y and Peng C: Overview of MicroRNA biogenesis, mechanisms of actions, and circulation. Front Endocrinol (Lausanne) 9: 402, 2018.
13. World Medical Association: World Medical Association Declaration of Helsinki: Ethical principles for medical research involving human subjects. JAMA 310: 2191-2194, 2013.

14. Livak KJ and Schmittgen TD: Analysis of relative gene expression data using real-time quantitative PCR and the 2(-Delta Delta C(T)) method. Methods 25: 402-408, 2001.

15. Li C, Li X, Gao X, Zhang R, Zhang Y, Liang H, Xu C, Du W, Zhang Y, Liu X, et al: MicroRNA-328 as a regulator of cardiac hypertrophy. Int J Cardiol 173: 268-276, 2014.

16. van Rooij E, Sutherland LB, Liu N, Williams AH, McAnally J, Gerard RD, Richardson JA and Olson EN: A signature pattern of stress-responsive microRNAs that can evoke cardiac hypertrophy and heart failure. Proc Natl Acad Sci USA 103: 18255-18260, 2006.

17. Verjans R, Peters T, Beaumont FJ, van Leeuwen $\mathrm{R}$, van Herwaarden T, Verhesen W, Munts C, Bijnen M, Henkens M, Diez J, et al: MicroRNA-221/222 family counteracts myocardial fibrosis in pressure overload-induced heart failure. Hypertension 71: 280-288, 2018.

18. Szczerba E, Zajkowska A, Bochowicz A, Pankiewicz K, Szewczyk G, Markiewicz K, Opolski G, Maciejewski T, Małecki M and Fijałkowska A: Rise in antifibrotic and decrease in profibrotic microRNA protect the heart against fibrosis during pregnancy: A preliminary study. Adv Clin Exp Med 27: 867-872, 2018.

19. Liang H, Zhang C, Ban T, Liu Y, Mei L, Piao X, Zhao D, $\mathrm{Lu}$ Y, Chu W and Yang B: A novel reciprocal loop between microRNA-21 and TGF $\beta$ RIII is involved in cardiac fibrosis. Int J Biochem Cell Biol 44: 2152-2160, 2012.

20. You XY, Huang JH, Liu B, Liu SJ, Zhong Y and Liu SM: HMGA1 is a new target of miR195 involving isoprenaline induced cardiomyocyte hypertrophy. Biochemistry (Moscow) 79: 538-544, 2014.

21. Heggermont WA, Papageorgiou AP, Quaegebeur A, Deckx S, Carai P, Verhesen W, Eelen G, Schoors S, van Leeuwen R, Alekseev S, et al: Inhibition of microRNA-146a and overexpression of its target dihydrolipoyl succinyltransferase protect against pressure overload-induced cardiac hypertrophy and dysfunction. Circulation 136: 747-761, 2017.

22. Feng B, Chen S, McArthur K, Wu Y, Sen S, Ding Q, Feldman RD and Chakrabarti S: miR-146a-mediated extracellular matrix protein production in chronic diabetes complications. Diabetes 60: 2975-2984, 2011.

23. Tao L, Bei Y, Zhang H, Xiao J and Li X: Exercise for the heart: Signaling pathways. Oncotarget 6: 20773-20784, 2015.

24. Liu X, Xiao J, Zhu H, Wei X, Platt C, Damilano F, Xiao C, Bezzerides V, Boström P, Che L, et al: miR-222 is necessary for exercise-induced cardiac growth and protects against pathological cardiac remodeling. Cell Metab 21: 584-95, 2015.

25. Yang S, Fan T, Hu Q, Xu W, Yang J, Xu C, Zhang B, Chen J and Jiang H: Downregulation of microRNA-17-5p improves cardiac function after myocardial infarction via attenuation of apoptosis in endothelial cells. Mol Genet Genomics 293: 883-894, 2018.

26. Ong J, Woldhuis RR, Boudewijn IM, van den Berg A, Kluiver J, Kok K, Terpstra MM, Guryev V, de Vries M, Vermeulen CJ, et al: Age-related gene and miRNA expression changes in airways of healthy individuals. Sci Rep 9: 3765, 2019.

This work is licensed under a Creative Commons Attribution 4.0 International (CC BY-NC 4.0) License 